\title{
Effectiveness and Safety of Dimethyl Fumarate Treatment in Relapsing Multiple Sclerosis Patients: Real-World Evidence
}

\author{
Raed Alroughani - Samar Farouk Ahmed - Raed Behbehani · \\ Jasem Al-Hashel
}

Received: June 27, 2017 / Published online: August 5, 2017

(C) The Author(s) 2017. This article is an open access publication

\section{ABSTRACT}

Introduction: Dimethyl fumarate (DMF) has been recently approved as a disease-modifying therapy for the treatment of multiple sclerosis (MS). Post-marketing studies are important to confirm what was established in clinical trials.

Objective: To evaluate effectiveness and safety of DMF and to measure the occurrence of lymphopenia in a cohort of MS patients in a clinical setting.

Methods: Using the national MS registry, we prospectively assessed relapsing MS patients

Enhanced content To view enhanced content for this article go to http://www.medengine.com/Redeem/ 88F8F0605789D87D.

R. Alroughani $(\bowtie)$

Division of Neurology, Department of Medicine,

Amiri Hospital, Sharq, Kuwait City, Kuwait

e-mail: raed.alroughani@dasmaninstitute.org

S. F. Ahmed · J. Al-Hashel

Department of Neurology, Ibn Sina Hospital, Sabah

Medical Area, Kuwait City, Kuwait

S. F. Ahmed

Department of Neurology and Psychiatry, Minia

University, Minia, Egypt

R. Behbehani

Department of Ophthalmology, Al-Bahar Eye

Center, Sabah Medical Area, Kuwait City, Kuwait

J. Al-Hashel

Department of Medicine, Faculty of Medicine,

Kuwait University, Jabriya, Kuwait who had been prescribed DMF for at least 6 months. Primary outcome measure was the proportion of relapse-free patients at last follow-up visit. Secondary outcome measures were the mean change in expanded disability status scale (EDSS) and the proportion of patients with radiological activity (gadolinium-enhancing or new T2 lesions) at the last follow-up visit. Absolute lymphocyte count (ALC) was assessed at baseline (within 6 months prior to DMF initiation) and at one or more times during DMF treatment 3 months post-initiation.

Results: Of 134 patients identified, 119 were eligible and included in the analysis. Women represented $59.7 \%$ of the studied cohort. Mean age and mean disease duration were $33.5 \pm 11.1$ and $8.3 \pm 7$ years, respectively. A total of $75.6 \%$ of the patients received prior disease-modifying therapies. Mean duration of DMF exposure was $20.5 \pm 9.5$ months. The proportion of relapse-free patients increased significantly from $51.2 \%$ to $89.9 \%(p<0.0001)$, while the mean EDSS score decreased from $2.8 \pm 1.8$ at baseline to $2.3 \pm 1.7(p<0.058)$ at last follow-up visit. The proportion of patients with MRI activity decreased significantly from $61.1 \%$ to $15.1 \%$ $(p<0.0001)$. The mean ALCs decreased from 2170 to 1430 cells $/ \mu$ l (34\% decrease). Lymphopenia was seen in $13(10.9 \%)$ patients, of whom $3(2.5 \%)$ patients had grade 3 lymphopenia necessitating discontinuation of DMF. Although no serious adverse events were reported, $19.3 \%$ of patients discontinued DMF. 
Conclusion: In clinical practice, DMF appeared to be effective in reducing disease activity and progression of disability throughout the observational period. DMF was well tolerated with no serious adverse events. ALC profiles in DMF-treated patients were generally stable throughout the observational period. The proportion of patients who developed severe lymphopenia was similar to figures in clinical trials.

Keywords: Dimethyl fumarate; Kuwait; Lymphopenia; Multiple sclerosis; Safety

\section{INTRODUCTION}

Multiple sclerosis (MS) is a chronic inflammatory demyelinating disease affecting more than 2.3 million people worldwide [1]. Dimethyl fumarate (DMF) has been approved as a disease-modifying therapy for the treatment of relapsing MS [2]. The proposed mechanism of action of DMF involves the activation of nuclear factor-related 2 (Nrf2) pathway resulting in augmenting the antioxidative capacities, and hence reducing inflammation [3]. Phase II and III clinical trials showed that DMF was effective in reducing both clinical and radiological disease activity [4-6]. Few adverse events were reported in phase III clinical trials that included lymphopenia (grade 3; absolute lymphocyte count <500), which developed in approximately $5 \%$ of patients $[5,6]$. We aimed to prospectively assess relapsing MS patients who were treated with DMF, in terms of effectiveness, tolerability, and safety in addition to the rate of occurrence of lymphopenia in a real clinical sitting.

\section{METHODS}

We conducted a prospective study utilizing the national MS registry to evaluate relapsing MS patients who were prescribed $240 \mathrm{mg}$ DMF twice a day. The registry was established in 2010 in Kuwait after combining the databases of all major hospitals [7]. All patients who were entered in the registry were followed prospectively; as per the registry protocol, patients had at least two scheduled visits per year. The treating neurologist evaluated patients at unscheduled visits if there were any new neurologic symptoms suggestive of relapse or if they had experienced side effects. Neurological examinations, including assessment of expanded disability status scale (EDSS), were performed every 6 months [8]. Magnetic resonance imaging (MRI) of the brain and cervical spine was obtained at baseline, biannually for the first year, and subsequently on an annual basis. Imaging was performed utilizing a standardized MS protocol.

Data of demographics (age, gender), clinical characteristics (course of the disease, disease duration, relapse rate, EDSS score), and treatment parameters [prior disease modifying therapies (DMTs) usage, DMF duration, adverse events (AEs)], MRI scans, and laboratory data of absolute lymphocyte count (ALC) were collected. Patients with incomplete data or progressive (primary or secondary) MS were excluded. A relapse was defined as new or recurrent neurologic symptom not associated with fever or infection that lasted for at least $24 \mathrm{~h}$ and was accompanied by new neurologic signs found by the examining neurologist. Disease progression was defined by an increase of at least 1 point in EDSS score in patients with EDSS less than 6 or a 0.5 -point increase in those with EDSS of at least 6 [9]. Confirmed disease progression was assessed at least 6 months after the initial EDSS score. EDSS measurements within 30 days of the relapse were excluded to avoid the bias of falsely elevated EDSS scores during a relapse. MRI activity was defined as the presence of new or enlarging T2 lesions and/or gadolinium-enhancing T1 lesions in a follow-up scan.

The primary outcome was to assess the proportion of relapse-free patients at last follow-up. Secondary outcome measures included the assessment of change in confirmed disease progression measured by EDSS scores, and the proportion of patients with radiological activity (gadolinium-enhancing or new T2 lesions) at the end of the observational period. Grades of lymphopenia were assigned according to the common terminology criteria for adverse events: grade 1, ALC of 800 to the lower limit of normal; grade 2, ALC 500-799; and grade 3 , ALC less than 500 [10]. 
All analyses were performed using SPSS 20 for Windows. Simple descriptive statistical tests (mean and standard deviation) were used to describe the numerical values of the sample. The significance of the differences of mean EDSS scores and number of relapses before and after treatment were compared by using the paired-sample Student's $t$ test, while $\chi^{2}$ tests were used for nonparametric variables (proportion of relapse-free patients and MRI activity); a $p$ value less than 0.05 was regarded as significant. The institutional ethical committee approved the study and all patients signed the informed consent forms.

\section{RESULTS}

A total of 134 relapsing-remitting multiple sclerosis (RRMS) patients were treated with DMF were included in the registry, of whom 119 patients were included in the analysis of efficacy. Eleven patients were excluded from the efficacy analysis because of short exposure to DMF of less than 6 months, while four patients were excluded because of incomplete data. Patients' demographics are outlined in Table 1. Women represented $59.7 \%$ of the studied cohort. Mean age and mean disease duration were $33.5 \pm 11$ years and $8.3 \pm 7$ years, respectively. The indications to prescribe DMF are presented in Table 1. Most of the analyzed patients $(n=90 ; 75.6 \%)$ were exposed to prior disease-modifying therapy (DMTs).

The mean duration of DMF exposure in the cohort was $20 \pm 9.5$ months. The proportion of relapse-free patients increased significantly from $51.2 \%$ to $89.9 \%(p<0.0001)$ at the end of observational period (Table 2). DMF was associated with a reduction of disability progression when baseline EDSS scores were compared to last follow-up visits $(2.8 \pm 1.8$ vs. $2.3 \pm 1.7$; $p<0.058)$. Few patients $(n=17 ; 14.3 \%)$ had improvement of their EDSS scores, while $(n=8$; $6.7 \%)$ showed progression of their disability. None of the patients who had improvement in their EDSS scores sustained a relapse, while five of eight patients who had EDSS worsening
Table 1 Demographic and clinical characteristics of the studied cohort $(n=119)$

\begin{tabular}{|c|c|}
\hline Variables & $\begin{array}{l}\text { Mean } \pm S D / \\
\text { number }(\%)\end{array}$ \\
\hline \multicolumn{2}{|l|}{ Gender } \\
\hline Female & $71(59.7)$ \\
\hline Male & $48(40.3)$ \\
\hline Mean age (years) & $33.5 \pm 11.1$ \\
\hline Range & $16-63$ \\
\hline Mean age of onset (years) & $25.2 \pm 8.4$ \\
\hline Range & $7-60$ \\
\hline Mean disease duration (years) & $8.3 \pm 7$ \\
\hline Range & $1-24$ \\
\hline $\begin{array}{l}\text { Mean duration of DMF exposure } \\
\text { (months) }\end{array}$ & $20.5 \pm 9.5$ \\
\hline Range & $6-44$ \\
\hline \multicolumn{2}{|l|}{ Indication to initiate DMF } \\
\hline Newly diagnosed RRMS & $29(24.4)$ \\
\hline Relapse & $28(23.5)$ \\
\hline Radiological activity & $13(10.9)$ \\
\hline Tolerability & $16(13.4)$ \\
\hline Convenience & $15(12.6)$ \\
\hline JCV seropositivity & $10(8.4)$ \\
\hline Adverse events to prior DMTs & $8(6.7)$ \\
\hline $\begin{array}{l}\text { Prior use of disease-modifying } \\
\text { therapies }\end{array}$ & $90(75.6)$ \\
\hline Interferon beta $1 \mathrm{~b}$ SC & $28(31.1)$ \\
\hline Interferon beta 1a IM & $26(28.9)$ \\
\hline Interferon beta 1a SC & $12(13.3)$ \\
\hline Fingolimod & $10(11.1)$ \\
\hline Teriflunomide & $4(4.4)$ \\
\hline Natalizumab & $10(11.1)$ \\
\hline
\end{tabular}

$D M F$ dimethyl fumarate, $S D$ standard deviation, $R R M S$ relapsing-remitting multiple sclerosis, JCV John Cunningham virus, $D M T s$ disease-modifying therapies 
Table 2 Primary and secondary outcome measures of the studied cohort $(n=119)$

\begin{tabular}{lll}
\hline Variables & $\begin{array}{l}\text { Baseline } \\
(\text { mean } \pm \text { SD)/ } \\
\text { number (\%) }\end{array}$ & $\begin{array}{l}\text { Last } \\
\text { follow-up } \\
(\text { mean } \pm \text { SD)/ } \\
\text { number (\%) }\end{array}$ \\
\end{tabular}

\begin{tabular}{llll}
\hline $\begin{array}{l}\text { Primary } \\
\text { Proportion }\end{array}$ & $61(51.3)$ & $107(89.9)$ & $<0.0001^{*}$ \\
of & & & \\
relapse-free & & & \\
patients & & & \\
Secondary & & $2.3 \pm 1.7$ & 0.058 \\
Mean EDSS & $2.8 \pm 1.8$ & & \\
score & & $18(15.1 \%)$ & $<0.0001^{*}$ \\
MRI activity & $74(61.1 \%)$ &
\end{tabular}

$S D$ standard deviation, EDSS expanded disability status scale, $M R I$ magnetic resonance imaging

*Statistically significant

sustained a relapse during the observational period. The proportion of patients with MRI activity decreased significantly from $61.1 \%$ to $15.1 \%(p<0.001)$ as outlined in Table 2.

The mean ALCs decreased by $34 \%$ from 2170 at baseline to 1430 cells/ $\mu$ l at last follow-up. Lymphopenia was seen in 13 (10.9\%) patients, of whom 10 (8.4\%) patients had grade 1-2 lymphopenia and 3 (2.5\%) patients had persistent grade III lymphopenia necessitating discontinuation.

A total of 47 (39.5\%) adverse events were registered; GI upset and flushing were the most common reported symptoms, while lymphopenia was the common most laboratory abnormality observed in the analyzed cohort (Table 3). Breakthrough disease activity was the most common reason for discontinuation. No major AEs or opportunistic infections occurred in the studied cohort.

\section{DISCUSSION}

Post-marketing studies are important to confirm what was established in clinical trials, and to assess the intermediate and long-term safety.
Table 3 Safety parameters of the study cohort $(n=119)$

\begin{tabular}{lc}
\hline Variables & Number (\%) \\
\hline Adverse events & $47(39.5)$ \\
Gastrointestinal upset & $15(12.6)$ \\
Flushing & $8(6.7)$ \\
Headache & $6(5)$ \\
Lymphopenia & $13(10.9)$ \\
Elevated liver transaminases & $3(2.5)$ \\
Others & $2(1.7)$ \\
Reason for discontinuation & $23(19.3)$ \\
Disease breakthrough & $14(11.8)$ \\
Clinical relapse & $12(10.1)$ \\
MRI activity & $2(1.7)$ \\
Persistent lymphopenia (grade 3$)$ & $3(2.5)$ \\
Gastrointestinal upset & $3(2.5)$ \\
Confirmed pregnancy & $2(1.7)$ \\
Elevated liver transaminases & $1(0.8)$ \\
\hline
\end{tabular}

$S D$ standard deviation, $M R I$ magnetic resonance imaging

We found that DMF was associated with improvements in both clinical and MRI outcome measures in patients with relapsing MS after a mean observational period of 20 months. Our results are in line with what previous phase III clinical trials, DEFINE and CONFIRM, had shown in that DMF was effective in reducing annualized relapse rate (ARR) and disease progression as measured by means of EDSS $[5,6]$. The proportion of relapse-free patients in our cohort was higher than the pivotal trials of DMT. Disability progression was reduced by $14 \%$ in our cohort, slightly lower than what was observed in pivotal trials (38\% in DEFINE and $21 \%$ in CONFIRM) $[5,6]$. This could be explained by the fact that approximately $30 \%$ of the patients were switched to DMF because of tolerability/AEs to previous DMTs or because of convenience and willingness to initiate oral medications rather than injectable ones. Additionally, most of the patients (ca. 40\%) who initiated DMF did not have any ongoing clinical disease activity, especially those who were 
switched from natalizumab due to JCV seropositivity. Moreover, approximately three-quarters of our patients were exposed to prior DMTs, which is higher than the $30-40 \%$ figures in DEFINE and CONFIRM trials $[5,6]$.

Few post-marketing observational studies have been published so far $[11,12]$. In a prospective study in a US community health center evaluating 412 patients over 3 years, $86 \%$ of patients were relapse free and $25 \%$ had activity MRI activity [11]. The proportion of relapse-free patients was similar to that in our cohort but the radiological activity was slightly higher than ours likely because of the inclusion of more natalizumab-treated patients $(n=44)$ in their cohort. A multicenter retrospective study from Germany which assessed longitudinal data of $644 \mathrm{MS}$ patients treated with DMF reported a $33 \%$ reduction in ARR from 0.52 to 0.35 at 1 year [12]; this was accompanied by a $33 \%$ reduction in EDSS progression from baseline of $0.15 \pm 0.49$ per year to $0.10 \pm 1.84$ per year during DMF treatment. Again, the demographic baseline differences especially in terms of age of the studied cohort, disease duration, and proportion of naïve patients $(45.2 \%)$ could explain the differences in efficacy when compared to our study.

Natalizumab discontinuation due to risk of progressive multifocal leukoencephalopathy (PML) is frequently seen in clinical practice especially after the introduction of the JC Stratify test [13]. In our cohort, of the 10 patients who were switched from natalizumab because of JCV seropositivity, three had radiological activities evident by the presence of new gadolinium-enhancing lesions, while one sustained a relapse within 4 months of initiation of DMF. This raises the potential risk of disease reactivation after transitioning from natalizumab. Several studies reported disease reactivation post-natalizumab discontinuation $[14,15]$. In a retrospective study that included 54 patients, the proportion of patients who experienced relapses was $57.4 \%$. Relapses occurred on average at 6.46 months (range 1-11 months). The ARR increased after natalizumab suspension $(p=0.0004)$, but it was significantly lower than the pre-natalizumab level $(p=0.0053)$. Similarly, more patients had new T2 lesions and new gadolinium-enhancing lesions after discontinuation of natalizumab $(p=0.0000)$. No rebound activity was observed. The institution of alternative therapies did not influence the increase in rate of disease reactivation, but the number was small and it was therefore difficult to draw any conclusion [14]. Similar findings were reported by another retrospective study conducted at two Italian MS referral centers which assessed patients who discontinued natalizumab up to 1 year after interruption. Of the 132 included patients, 72 patients $(54.5 \%)$ had relapses and 60 of 125 patients (48\%), who had MRI follow-ups, had radiological reactivation. Rebound was observed in 28 of 132 patients (21.2\%). Ninety-five patients $(72 \%)$ were switched to other therapies after a median washout period of 5 months. A lower risk of relapses was found in patients treated with second-line therapies (fingolimod) or off-label therapy (e.g., rituximab) than in those treated with first-line therapies (IFN-beta, GA, teriflunomide, azathioprine) [16]. The duration of washout period has been consistently observed in multiple studies to be a major factor for disease reactivation [17-19]. Most experts often prefer a short washout period (4-8 weeks) in order to avoid such risk. The risk of relapse after natalizumab cessation and switch to DMF is unknown. A few studies reported data on such cohorts, but the findings were limited by the small number of patients and short follow-up durations [20, 21]. Zurawski et al. assessed 30 patients treated with natalizumab for at least 12 months and then switched to DMF at a mean of 50 days. Five patients (17\%) suffered severe relapses, while $35 \%$ of patients had new lesions according to MRI activity. Relapses occurred at a mean of 3.5 months after natalizumab cessation [20]. Despite these findings, DMTs were associated with delayed long-term disability in MS patients. In an observational study on $3060 \mathrm{MS}$ patients, the risks of attaining EDSS 3.0 were $94 \%$ and $73 \%$ lower in patients treated with immunomodulators and immunosuppressants, respectively, compared to untreated patients. However, the risk of attaining EDSS 6.0 was higher in patients starting immunomodulators after EDSS 3.0 than before $(\mathrm{HR}=4.42)$ [22]. 
Thus, even in patients with longer disease duration or with neurological disabilities, there is a window of opportunity to delay disease progression in the long term.

Most of the observed AEs in our cohort were transient and managed either with dose reduction for 2-4 weeks or symptomatic therapies (e.g., aspirin for flushing). Gastrointestinal upset and flushing were the most common adverse events and these were known to be most prominent within the first few months of initiating therapy $[5,6,11]$. Although $19.3 \%(n=23)$ of our patients discontinued DMF treatment, this is lower than the $30 \%$ discontinuation rates reported in the pivotal trials $[5,6]$ and the 29-38\% discontinuation rate in other observational studies [11, 12]. Nevertheless, ongoing disease activity was the main reason for discontinuation across all studies. The rate of discontinuation due to GI upset was slightly lower in our cohort than what was reported in the pivotal studies $(1.7 \%$ versus $7 \%)[5,6]$. Similarly, in an observational study assessing the GI tolerability in 233 patients taking DMF for up to 12 weeks, $7.3 \%$ of patients discontinued DMF because of gastrointestinal AEs [23]. The could be explained by the reporting bias since patients were prompted to report onset and offset times of any AEs including GI-related events in controlled studies. Another explanation for our low discontinuation rate secondary to GI upset is the fact that we adopted a protocol of titrating the DMF dose over 4 weeks (compared to the standard 1-week titration) and prescribed antacids and proton pump inhibitors earlier when symptoms started.

Most of the observed grade 1 and 2 lymphopenia were transient in keeping with what was reported in DEFINE and CONFIRM $[5,6]$. The reported grade 3 lymphopenia in our study is consistent with figures of other post-approval studies [24-26]. Moreover, the rate of lymphopenia necessitating discontinuation of DMF in our cohort $(2.5 \%)$ was similar to the $4-5 \%$ rates reported in other studies but lower than the reported $11 \%$ in the observation US study which included older patients (mean age $49 \pm 12$ years). Lymphopenia presumably reflects some degree of immune suppression in DMF-treated patients, and there is therefore a theoretical basis for supposing that these patients would be less likely to experience breakthrough MS activity [26]. The mechanism of DMF-induced lymphopenia is not known. In vitro studies suggest that it may induce $T$ cell apoptosis [27].

There are several limitations in our study. First, it is an open-label with no placebo arm resulting in methodological bias. Second, more than half of the studied cohort had no disease activity when they started DMF. It should be noted that in our practice, DMF is used primarily used as a first-line therapy, mostly including newly diagnosed naïve patients or those who had tolerability issues with injectable DMTs. Patients with highly active disease (at least two relapses in 1 year) or have aggressive presentation at onset (i.e., accumulating neurological disabilities) are usually stratified to second-line therapies such as fingolimod, natalizumab, or alemtuzumab. Hence, the results should be interpreted with caution and more importantly should not be generalized. Third, most of the newly diagnosed patients in our study did not have sufficient follow-up time prior to DMF exposure as treatment is usually started within 6 months of diagnosis. Subsequently, the annual relapse rate prior to DMF initiation could not be measured in order to report post-DMF ARR. Nevertheless, our study was one of the few prospective studies and the first to be performed in a region that has a relatively adequate length of DMT exposure.

\section{CONCLUSION}

Dimethyl fumarate was associated with a significant reduction in clinical and radiological disease activity. During the observational period, DMF appeared to be safe and tolerable. Few patients developed grade 3 lymphopenia. Our results were in parallel with what was seen in pivotal trials.

\section{ACKNOWLEDGEMENTS}

The authors thank the study participants for their involvement in the study and also thank 
the administrative assistants at Amiri and Ibn Sina Hospitals for their continuous support. No funding sponsorship was received for this study or publication of this article. The article processing charges were funded by the authors.

Disclosures. Dr. Read Alroughani received honoraria as a speaker and for serving on scientific advisory boards from Bayer, Biogen, Novartis, Sanofi-Genzyme, Roche, and Merck. Drs. Samar Farouk Ahmed, Read Behbehani, and Jasem Al-Hashel have nothing to disclose.

Compliance with Ethics Guidelines. All procedures followed were in accordance with the ethical standards of the responsible committee on human experimentation (institutional and national) and with the Helsinki Declaration of 1964, as revised in 2013. Informed consent was obtained from all patients for being included in the study.

Open Access. This article is distributed under the terms of the Creative Commons Attribution-NonCommercial 4.0 International License (http://creativecommons.org/licenses/ by-nc/4.0/), which permits any noncommercial use, distribution, and reproduction in any medium, provided you give appropriate credit to the original author(s) and the source, provide a link to the Creative Commons license, and indicate if changes were made.

Author Contributions All named authors meet the International Committee of Medical Journal Editors (ICMJE) criteria for authorship for this manuscript, take responsibility for the integrity of the work as a whole, and have given final approval for the version to be published.

\section{REFERENCES}

1. Tullman MJ. Overview of the epidemiology, diagnosis, and disease progression associated with multiple sclerosis. Am J Manag Care. 2013;19:S15-20.
2. FDA. Prescribing information: TECFIDERA. 2013. http://www.accessdata.fda.gov/drugsatfda_docs/ label/2013/204063lbl.pdf. Accessed June 12, 2017.

3. Scannevin RH, Chollate S, Jung MY, et al. Fumarates promote cytoprotection of central nervous system cells against oxidative stress via the nuclear factor (erythroid-derived 2)-like 2 pathway. J Pharmacol Exp Ther. 2012;341:274-84.

4. Kappos L, Gold R, Miller DH, et al. Efficacy and safety of oral fumarate in patients with relapsing-remitting multiple sclerosis: a multicentre, randomised, double-blind, placebo-controlled phase IIb study. Lancet. 2008;372:1463-72.

5. Fox RJ, Miller DH, Phillips JT, et al. Placebo-controlled phase 3 study of oral BG-12 or glatiramer in multiple sclerosis. N Engl J Med. 2012;367:1087-97.

6. Gold R, Kappos L, Arnold DL, et al. Placebo-controlled phase 3 study of oral BG-12 for relapsing multiple sclerosis. $\mathrm{N}$ Engl J Med. 2012;367:1098-107.

7. Alroughani R, Ahmed SF, Behbahani R, et al. Increasing prevalence and incidence rates of multiple sclerosis in Kuwait. Mult Scler. 2014;20:543-7.

8. Kurtzke JF. Rating neurologic impairment in multiple sclerosis: an expanded disability status scale (EDSS). Neurology. 1983;33:1444-52.

9. Lublin FD, Baier M, Cutter G. Effect of relapses on development of residual deficit in multiple sclerosis. Neurology. 2003;61:1528-32.

10. Publication N. National Cancer Institute. Common terminology criteria for adverse events. 2009;4: \#09-7473.

11. Smoot K, Spinelli KJ, Stuchiner T, et al. Three-year clinical outcomes of relapsing multiple sclerosis patients treated with dimethyl fumarate in a United States community health center. Mult Scler. 2017. doi:10.1177/1352458517709956.

12. Miclea A, Leussink VI, Hartung HP, Gold R, Hoepner R. Safety and efficacy of dimethyl fumarate in multiple sclerosis: a multi-center observational study. J Neurol. 2016;263:1626-32.

13. Lee P, Plavina T, Castro A, et al. A second-generation ELISA (STRATIFY JCV DxSelect) for detection of JC virus antibodies in human serum and plasma to support progressive multifocal leukoencephalopathy risk stratification. J Clin Virol. 2013;57:141-6.

14. Melis M, Cocco E, Frau J, et al. Post-natalizumab clinical and radiological findings in a cohort of 
multiple sclerosis patients: 12-month follow-up. Neurol Sci. 2014;35:401-8.

15. Lo Re M, Capobianco M, Ragonese P, et al. Natalizumab discontinuation and treatment strategies in patients with multiple sclerosis (MS): a retrospective study from two Italian MS centers. Neurol Ther. 2015;4:147-57.

16. Vidal-Jordana A, Tintore M, Tur C, et al. Significant clinical worsening after natalizumab withdrawal: predictive factors. Mult Scler. 2015;21:780-5.

17. de Seze J, Ongagna JC, Collongues N, et al. Reduction of the washout time between natalizumab and fingolimod. Mult Scler. 2013;19:1248.

18. Jokubaitis VG, Li V, Kalincik T, et al. Fingolimod after natalizumab and the risk of short-term relapse. Neurology. 2014;82:1204-11.

19. Kerbrat A, Le Page E, Leray E, et al. Natalizumab and drug holiday in clinical practice: an observational study in very active relapsing remitting multiple sclerosis patients. J Neurol Sci. 2011;308:98-102.

20. Zurawski J, Flinn A, Sklover L, Sloane JA. Relapse frequency in transitioning from natalizumab to dimethyl fumarate: assessment of risk factors. J Neurol. 2016;263:1511-7.

21. Patti F, Leone C, Zappia M. Clinical and radiologic rebound after discontinuation of natalizumab therapy in a highly active multiple sclerosis patient was not halted by dimethyl-fumarate: a case report. BMC Neurol. 2015;15:252.

22. Cocco E, Sardu C, Spinicci G, et al. Influence of treatments in multiple sclerosis disability: a cohort study. Mult Scler. 2015;21:433-41.

23. Fox EJ, Vasquez A, Grainger W, et al. Gastrointestinal tolerability of delayed-release dimethyl fumarate in a multicenter, open-label study of patients with relapsing forms of multiple sclerosis (MANAGE). Int J MS Care. 2016;18:9-18.

24. Berkovich R, Weiner LP. Effects of dimethyl fumarate on lymphocyte subsets. Mult Scler Relat Disord. 2015;4:339-41.

25. Khatri BO, Garland J, Berger J, et al. The effect of dimethyl fumarate (Tecfidera) on lymphocyte counts: a potential contributor to progressive multifocal leukoencephalopathy risk. Mult Scler Relat Disord. 2015;4:377-9.

26. Longbrake EE, Cross AH. Dimethyl fumarate associated lymphopenia in clinical practice. Mult Scler. 2015;21:796-7.

27. Treumer F, Zhu K, Glaser R, Mrowietz U. Dimethylfumarate is a potent inducer of apoptosis in human $\mathrm{T}$ cells. $\mathrm{J}$ Invest Dermatol. 2003;121:1383-8. 